and their etiology. In particular, the study used the interpersonal negotiation strategies (INS) model of social problem solving, alongside a measure of self-schema, to investigate potential maladaptive social-cognitive processes in BPD. Empirical findings regarding schema domains specific to BPD are limited and research into cognitive processes has focused on the single concept of dichotomous thinking.

Methods: Participants were 30 youths (15-24 years) with three or more BPD traits and 30 youths (15-24 years) with MDD. Participants received self-report measures of attachment, self-schema and interpersonal functioning. The INS interview assessed social perspective coordination skills in response to six hypothetical vignettes depicting situations of interpersonal conflict. To investigate the parameters of social information-processing biases, in $\mathrm{BPD}$, vignettes varied in terms of whether the conflict resembled BPD-specific schema content or neutral-schema content.

Results: The BPD group scored significantly higher on schemas of mistrust/abuse, insufficient self-control and entitlement and were characterized by high attachment anxiety and avoidance, but were differentiated from the MDD group by higher avoidance. The BPD group responded to interpersonal conflict with social perspective coordination skills of a significantly lower developmental level than the MDD group, regardless of vignette content. Young people with BPD traits were accordingly characterized by significantly worse interpersonal functioning.

Conclusion: The implications of these results for early cognitive intervention approaches to the treatment of $\mathrm{BPD}$ are discussed.

02-05

\section{The relationship of self-structure, attachment style and rejection sensitivity to borderline personality features}

\section{Hulbert, J Boldero, R Bell}

School of Behavioural Science, The University of Melbourne, Melbourne, Australia

Background: Views of self, others, relationships and social functioning form a significant part of the clinical picture of borderline personality disorder (BPD). Theoretical models have variously implicated temperament, most notably neuroticism, and aberrant attachment experience, along with related deficits in sense of self and social cognition in the etiology of this serious mental disorder. However, no research has systematically investigated the extent to which these views are associated with the characteristics of BPD. The present study examines the relationship of self-complexity and self-discrepancy, attachment style and rejection sensitivity to endorsement of BPD features.

Method: As part of course requirements, 101 first-year university students (70 women and 31 men) completed well-validated self-report measures of neuroticism, self-complexity and self-discrepancy, attachment style, rejection sensitivity and an 80 -item screening measure for BPD.

Results: With levels of neuroticism controlled for, significant positive relationships were found between attachment anxiety and avoidance, rejections sensitivity and endorsement of BPD features. In addition, the extent to which participants saw themselves as discrepant from their ideal and ought self-guides [actualideal (AI) and actual-ought (AO) self-discrepancies] in terms of both positive and negative self-roles and attributes were assessed, as was the extent to participants' self-concepts were complex with regard to the organization of positive and negative attributes. Reporting more BPD features was associated with large negative $\mathrm{AI}$ and $\mathrm{AO}$ discrepancies (reflecting greater possession of negative traits), as were the complexity of both negative roles and attributes. The data suggest that both the self-concept content and structure contribute to BPD.

Conclusion: The theoretical and clinical implications of the present findings and planned future research into the role of self and social cognition in BPD are discussed.

02-06

\section{Personality disorder in the elderly and the effect on outcome of comorbid axis I disorder}

\section{J Stevenson}

Westmead and Cumberland Hospitals and Sydney University, Sydney, Australia

Personality disorder in younger adult psychiatric inpatients is known to prolong the in-patient stay, impede recovery and increase health care costs. There is a significant association between the number of disordered personality traits and medical resource utilization. However, this has not been extensively studied in the elderly, where personality disorder is usually undiagnosed or ignored. It has previously been felt that personality disorder 'burns out' in old age, but there is no data supporting this. Our study is to determine the prevalence and types of personality disorder in elderly psychiatric in-patients and the impact if any it has 
on their ultimate outcome. Previous work has found that a high percentage of older psychiatric in-patients $(50 \%-60 \%)$ had a personality disorder. Others have also confirmed a poorer outcome of axis I disorders in personality-disordered clients. However, this could vary widely resulting from different sample characteristics, different measures and different diagnostic criteria. The presence of personality disorders has also been shown to adversely affect relationships, which may also be deleterious to recovery, as supportive relationships improve/hasten recovery. Our hypothesis is that those elderly psychiatric in-patients with comorbid personality disorders will take longer to treat, and their recovery will be less complete than those without personality disorders. A single case series study of adult psychiatric in-patients is being undertaken to determine the prevalence of personality disorder and the effect on treatment of their axis I disorder. This study will contribute to our knowledge of the effects of personality disorder in elderly psychiatric in-patients and should provide clues as to how these conditions could be managed to affect improved outcomes.

02-07

\section{The effect of personality disorder on treatment for depression: psychotherapy vs. medication}

\section{RT Mulder, PR Joyce, SE Luty, CM Frampton, JD Carter}

Department of Psychological Medicine, Christchurch School of Medicine \& Health Sciences, University of Otago, Christchurch, New Zealand

Aims: To study the impact of comorbid personality disorders (PDs) on outcome in 1) a medication trial for major depression (MD) and 2) a psychotherapy trial for MD.

Method: Two treatment trials for patients with MD were performed: 1) randomized clinical trial of fluoxetine vs. nortriptyline with open follow-up $(n=195)$ and 2) randomized clinical trial of interpersonal psychotherapy (IPT) vs. cognitive behavioural therapy $(n=177)$.

Results: Comorbid PD had no impact on the outcome of MD in patients treated with medication over the short term. Avoidant PD was associated with poorer outcome over the longer term. Comorbid PD had a negative impact on the outcome of MD in patients treated with psychotherapy over the short term. This was largely explained by the poor response to IPT in depressed patients with comorbid PD.
Discussion: The finding that comorbid PD adversely affects the outcome in depressed patients treated with psychotherapy but not with drugs is consistent with other studies. Possible mechanisms are discussed.

02-08

\section{A randomized controlled trial of psychotherapeutic early intervention for borderline personality disorder}

\author{
A Chanen ${ }^{1,2}$, H Jackson ${ }^{1,3}$, L McCutcheon ${ }^{1,2}$, \\ D Germano ${ }^{2}$, H Nistico², P Dudgeon ${ }^{3}$, HP Yuen', \\ E McDougall', C Weinstein', V Clarkson', P McGorry ${ }^{1,2}$
}

'ORYGEN Research Centre, Department of Psychiatry, The University of Melbourne; ${ }^{2}$ ORYGEN Youth Health, Melbourne Health; and ${ }^{3}$ School of Behavioural Science,

The University of Melbourne, Melbourne, Australia

Background: Borderline personality disorder (BPD) is a major public health problem. This study aims to delay the onset of or reduce the severity of subsequent BPD in a sample of adolescents at risk of BPD.

Method: Eighty-six 15- to 18-year-old participants were drawn from an adolescent psychiatric service. They had one or more childhood risk factors for BPD and met criteria for two or more current DSM-IV BPD features, using rigorous diagnosis. Participants were randomly allocated to 24 sessions of either cognitive analytic therapy [CAT; Ryle 1997] or manualized 'good clinical care' (GCC). A third, nonrandomized comparison group $(n=30)$, which received 'treatment as usual' (TAU), was collected prior to implementation of the study. Outcome measures completed at baseline, 6, 12 and 24 months included BPD score, internalizing and externalizing psychopathology and social and occupational functioning.

Results: At 24 months, patients receiving CAT had statistically significant lower levels of externalizing pathology compared with those receiving GCC. Patients receiving CAT had statistically significant lower scores on internalizing and externalizing psychopathology compared with those receiving TAU, and patients receiving GCC had statistically significant lower scores on internalizing psychopathology compared with TAU. BPD scores improved from baseline levels in all three conditions and no significant differences were found between the groups.

Conclusion: CAT is an effective early intervention for BPD and is superior to both manual-based ' $\mathrm{GCC}$ ' and TAU. 